- Original Article

\title{
BCL11A rs1427407 Genotypes in Sickle Cell Anemia Patients Undergo to Stroke Problems in Sudan
}

\author{
Fathelrahman Mahdi Hassan*, Faisal Mousa Al-zahrani \\ Department of Clinical Laboratory Science, College of Applied Medical Science, Imam Abdulrahman Bin Faisal University, Dammam, Saudi Arabia
}

Background: Sickle cell disease is an autosomal recessive condition that results from the presence of a mutated form of hemoglobin. Some genetic variants of BCL11A are amenable to therapeutic manipulation. The present study investigated the relationship of a BCL11A variant (rs1427407) and its plasma levels with vaso-occlusive crises and stroke complications among patients in Sudan with sickle cell disease.

Methods: This cross-sectional study was performed between June 2014 and October 2016. The subjects included 166 patients who were diagnosed with sickle cell disease and 35 healthy control subjects, who were grouped according to sex and age ( $<15$ years, 15-25 years, and $>25$ years). All patients and/or their guardians provided informed consent. Blood samples were collected from the patients and controls under aseptic conditions.

Results: Plasma BCL11A levels were elevated in cases with vaso-occlusive crises that lasted for $>3$ years. In addition, plasma BCL11A levels were high in cases with the GG genotype (vs. GT and TT) at rs1427407. Furthermore, the BCL11A rs1427407 GG/GT genotypes increased the risk of vaso-occlusive crisis and stroke in the patients with sickle cell disease.

Conclusion: The BCL11A variant (rs1427407) and its plasma levels were associated with vaso-occlusive crisis and stroke in patients with sickle cell disease.

Keywords: BCL11A Protein; Genotype; Stroke; Sickle Cell Anemia; Sudan

Received: December 29, 2017, Revised: March 9, 2018, Accepted: March 16, 2018

${ }^{*}$ Corresponding Author: Fathelrahman Mahdi Hassan https://orcid.org/0000-0002-2630-3208

Tel: +966-530290049, Fax: +966-133330333, E-mail: fathmaga@yahoo.com, fmGameel@iau.edu.sa 


\section{INTRODUCTION}

Sickle cell disease (SCD) is an autosomal recessive condition that results from the presence of a mutated form of hemoglobin. It is the most common inherited blood disorder in the United Kingdom, where it affects 12,000-15,000 people among approximately 250,000 carriers of the sickle cell gene. ${ }^{1)}$ Globally, SCD is most common among people of West African or Caribbean descent. ${ }^{2)}$ The cause of SCD is a single nucleotide substitution in the beta globin gene on the short arm of chromosome 11. The resulting protein, hemoglobin S, polymerizes under low oxygen conditions, causing red blood cells to become rigid and sickle-shaped. These sickle cells cause the activation of other circulating cells, including neutrophils, and blood vessel walls as part of processes similar to those observed in vasculopathies. The tissue subsequently loses its blood supply, and the resulting ischemia causes tissue infarction. ${ }^{3)}$ The most common manifestations are painful vasoocclusive crisis (VOC), hemolytic anemia, and end organ damage caused by vasculopathy and tissue ischemia. Complications may involve a sudden onset, which is known as a sickle cell crisis, although some degree of sickling typically also leads to long-term organ damage. The VOC results from obstruction of the microvasculature by sickled red blood cells, which causes ischemia and pain. Crises may occur for no apparent reason, or there may be triggering a factor. Common triggering factors include dehydration, a sudden change in body temperature (e.g., caused by infection or environmental factors), or hypoxia caused by stress or exercise. ${ }^{4)}$ Fetal hemoglobin ( $\mathrm{HbF}$ ) levels are highly variable and inheritable, primarily through genetic variants at three principal loci: $B C L 11 A, H B S 1 L-M Y B$, and the $H B B$ cluster, which account for $10 \%-20 \%$ of $\mathrm{HbF}$ variations among SCD patients. ${ }^{5,6)}$ Some variations in $B C L 11 A$ are amenable to therapeutic manipulation, which led to the reversal of SCD symptoms in mouse models. ${ }^{7)}$ Func- tional studies have shown that the expression of BCL11A (a transcriptional repressor) is regulated by erythroid-specific enhancers that contain 3' DNase hypersensitive sites (DHS) located +62, + 58, and +55 kb from the transcription initiation site of $B C L 11 A{ }^{8)}$ Two single-nucleotide polymorphisms (SNPs) in the enhancer element have a strong association with $\mathrm{HbF}$ levels among African American SCD patients: rs1427407 in DHS +62 and rs7606173 in DHS +55. This is consistent with the hypothesis that multiple functional SNPs act in combination to influence $B C L 11 A$ regulation. ${ }^{5,8,9)}$ The present study investigated the relationship between a variant of BCL11A (rs1427407) and its plasma levels during VOC and stroke complications among SCD patients from Khartoum state, Sudan. The findings of this study may improve our ability to judge the severity of the sickle cell anemia (SCA), which can in turn help to improve its clinical management.

\section{METHODS}

This cross-sectional study was performed between June 2014 and October 2016 in the Omdurman State Teaching Hospital, Sudan. The study included 166 patients who were diagnosed with SCD or been hospitalized for treatment of painful VOC during the 6 months before the study. The analysis of VOC was restricted to subjects with a confirmed diagnosis of SCA. The patients were grouped according to sex and age group ( $<15$ years, $15-25$ years, and $>25$ years). In addition, 35 healthy subjects were recruited to create a control group with similar age and sex characteristics. The subjects and/or their guardians provided informed consent to participate in the study after receiving a detailed explanation regarding the purpose of the study and any related risks. Blood samples were collected from the patients and controls under aseptic conditions using an ethylenediaminetetraacetic acid-coated vacutainer tube.

Table 1. General characteristics of sickle cell anemia patients and controls

\begin{tabular}{|c|c|c|c|}
\hline Characteristic & Controls $(n=35)$ & Patients $(n=166)$ & P-value* \\
\hline Age (y) & & & 0.924 \\
\hline$<15$ & $8(22.8)$ & $33(19.9)$ & \\
\hline $15-25$ & $17(48.6)$ & $84(50.6$ & \\
\hline$>25$ & $10(28.6)$ & $49(29.5)$ & \\
\hline Sex & & & 0.432 \\
\hline Male & $19(54.3)$ & $102(61.4)$ & \\
\hline Female & $16(45.7)$ & $64(38.6)$ & \\
\hline \multicolumn{4}{|l|}{ Laboratory test results } \\
\hline Hemoglobin $(\mu \mathrm{g} / \mathrm{L})$ & $13.31 \pm 1.25$ & $12.02 \pm 4.66$ & 0.002 \\
\hline Red blood cell $(\mu \mathrm{g} / \mathrm{L})$ & $4.55 \pm 1.22$ & $4.32 \pm 2.17$ & 0.392 \\
\hline Mean corpuscular volume (fL) & $80.21 \pm 8.25$ & $80.33 \pm 11.40$ & 0.943 \\
\hline Mean corpuscular hemoglobin (pg) & $23.44 \pm 7.25$ & $24.35 \pm 9.82$ & 0.529 \\
\hline White blood cell count $(\mu \mathrm{g} / \mathrm{L})$ & $6.71 \pm 2.25$ & $11.22 \pm 3.91$ & $<0.05$ \\
\hline Platelets $(\mu \mathrm{g} / \mathrm{L})$ & $323.50 \pm 10.25$ & $368.27 \pm 143.46$ & $<0.05$ \\
\hline Fetal hemoglobin (\%) & $1.8 \pm 5.29$ & $20.25 \pm 7.28$ & $<0.05$ \\
\hline Reticulocytes (\%) & $5.2 \pm 3.65$ & $11.5 \pm 2.51$ & $<0.05$ \\
\hline BCL11A plasma level ( $\mu \mathrm{g} / \mathrm{L})$ & $70.88 \pm 3.25$ & $100.13 \pm 4.59$ & $<0.05$ \\
\hline
\end{tabular}

Values are presented as number (\%) or mean \pm standard deviation.

*Differences were considered statistically significant at $\mathrm{P}<0.05$ based on the $\mathrm{t}$-test or Fischer's exact test, as appropriate. 
A complete blood count was performed using a hematology analyzer (Sysmex XP-300/XP-100; Sysmex Asia Pacific Pte Ltd., Singapore). Levels of $\mathrm{HbF}$ were measured using high-performance liquid chromatography (BIO-RAD, Hercules, CA, USA). Plasma BCL11A levels were determined using an enzyme-linked immunosorbent assay (FY0208A; Hu Feng Biological Technology, Shanghai, China). The patients' peripheral blood samples were used to collect DNA samples according to the manufacturer's instructions (Puregene blood package; Qiagen, Germantown, MD, USA). Molecular analysis to detect the sickle mutation was performed using $200 \mathrm{ng}$ DNA and polymerase chain reaction (PCR) amplification of the $\beta$-globin gene with the appropriate oligonucleotide primers (sense: 5'-CACTGAACC CCCCACCTACCA-3', antisense: 5'-CTCCACTCCCCGTACCTTCC-3'). Direct sequencing was performed using the ABI prism 3730XL DNA sequencer (Applied Biosystems, Foster City, CA, USA), based on DDE I digestion of the PCR product. ${ }^{5,10)}$ Multiplex snap PCR and capillary electrophoresis were used to determine the rs 1427407 genotype. ${ }^{11)}$

All statistical analyses were performed using IBM SPSS ver. 19.0 (IBM Corp., Armonk, NY, USA). Results were expressed as mean \pm standard deviation and were compared between the patients and controls using the t-test or Fisher's exact test. Differences were considered statistically significant at P-values of $<0.05$. The allele frequency was calculated based on the different genotype distributions.

\section{RESULTS}

The characteristics and laboratory test results for the SCD patients and controls are shown in Table 1. There were no significant differences between the two groups according to age $(\mathrm{P}=0.9239)$ or $\operatorname{sex}(\mathrm{P}=0.4316)$. In addition, no significant differences were observed between the 116 SCD patients and the 35 controls according to mean red blood cell count $(\mathrm{P}=0.3916)$, mean corpuscular volume $(\mathrm{P}=0.9426)$, or mean corpuscular hemoglobin level $(\mathrm{P}=0.5288)$. However, there were significant

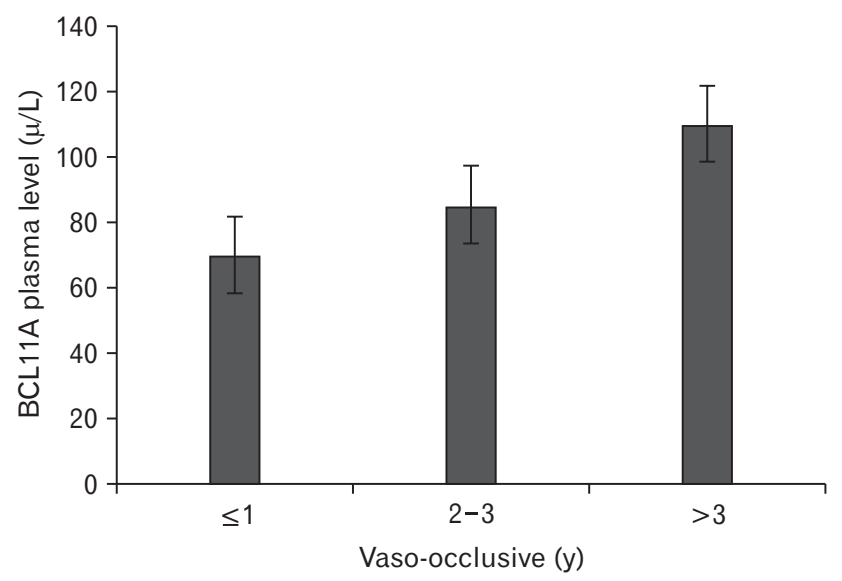

Figure 1. Plasma BCL11A levels and vaso-occlusive durations in sickle cell anemia patients. Vaso-occlusive crisis durations were divided into three groups: $\leq 1$ year, $2-3$ years, and $>3$ years. The BCL11A plasma level was increased in the group with $>3$ years of crisis. differences in the mean white blood cell count $(\mathrm{P}=0.0001)$, platelet count $(\mathrm{P}=0.0001)$, HbF percentage $(\mathrm{P}=0.0001)$, reticulocyte count $(\mathrm{P}=0.0001)$, and plasma BCL11A level $(\mathrm{P}=0.0001)$.

The rs1427407 sequencing revealed three genotypes: GG, GT, and TT. The distributions of the genotypes among the SCD patients and healthy controls are shown in Table 2 . Our results have been verified by the fact that SCD is associated with the BCL11A genotype polymorphism. The BCL11A genotypes that included the $\mathrm{T}$ allele and the $\mathrm{G}$ allele at rs1427407 were common among SCD patients. We evaluated whether SCD complications were associated with the various genotypes, and found that the BCL11A rs1427407 GG and GT genotypes had significantly elevated risks of SCD complications $(\mathrm{P}=0.0008)$ (Table 2).

The plasma BCL11A levels were measured in SCD patients according to their VOC duration (Figure 1), which revealed that plasma BCL11A levels were elevated among patients with a VOC duration of $>3$ years. In addition, plasma BCL11A levels were higher in patients with the GG genotype than in patients with the GT and TT genotypes (Figure 2). The frequencies of the BCL11A rs1427407 genotypes according to SCD complications are shown in Table 3 . We found that the

Table 2. The frequency of the BCL11A rs1427407 genotypes and alleles in the sickle cell anemia patients

\begin{tabular}{lccc}
\hline \multicolumn{1}{c}{ Variable } & Controls & Patients & P-value $^{\star}$ \\
\hline Genotype frequency & & & $<0.05$ \\
GG & $20(51.2)$ & $132(79.5)$ & \\
GT & $13(37.1)$ & $25(15.1)$ & \\
TT & $2(5.7)$ & $9(5.4)$ & \\
Total & $35(100.0)$ & $166(100.0)$ & \\
Allele frequency & & & $<0.05$ \\
G & $53(75.7)$ & $289(87.0)$ & \\
T & $17(24.3)$ & $43(13.0)$ &
\end{tabular}

Values are presented as number (\%).

*Differences were considered statistically significant at $\mathrm{P}<0.05$ based on Fisher's exact test.

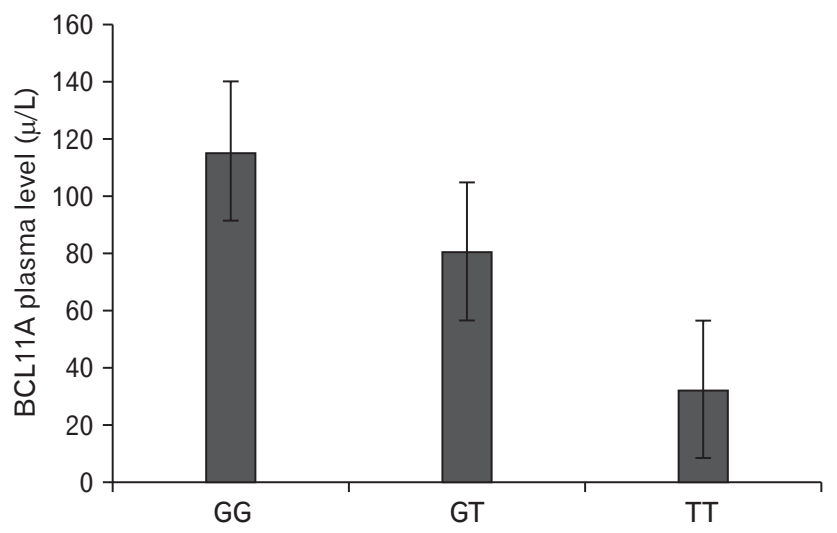

No. of genotype frequency (\%)

Figure 2. Association of plasma BCL11A levels with genotype frequencies in sickle cell anemia patients. The BCL11A genotypes were GG, GT, and TT. The BCL11A plasma level was associated with the GG genotype. 
Table 3. The frequency of BCL11A rs1427407 genotypes associated with VOC, stroke complications, and reticulocytes in sickle cell anemia patients

\begin{tabular}{lccrr}
\hline \multicolumn{1}{c}{ Variable } & GG & GT & $\Pi$ P & -value $^{*}$ \\
\hline VOC (y) & & & & $<0.05$ \\
$\leq 1$ & $4(3.4)$ & $16(19.8)$ & $9(27.3)$ & \\
$2-3$ & $22(19.0)$ & $21(26.0)$ & $13(39.4)$ & \\
$>3$ & $90(77.6)$ & $44(54.2)$ & $11(33.3)$ & \\
Stroke complications & & & & $<0.05$ \\
$\quad$ Recurrent & $86(74.1)$ & $41(50.6)$ & $19(57.6)$ & \\
$\quad$ Silent & $23(19.9)$ & $26(32.1)$ & $8(24.2)$ & \\
$\quad$ Predictors & $7(6.0)$ & $14(17.3)$ & $6(18.2)$ & \\
Reticulocytes (\%) & $116(100.0)$ & $76(93.8)$ & $28(84.8)$ & $<0.05$ \\
\hline
\end{tabular}

Values are presented as number (\%).

VOC, vaso-occlusive crisis.

${ }^{*}$ Differences were considered statistically significant at $\mathrm{P}<0.05$ based on Fisher's exact test.

GG, GT, and TT genotypes had significantly different VOC durations $(\mathrm{P}=0.0004)$ and significantly different rates of stroke $(\mathrm{P}=0.008)$ (Table 3). Thus, the BCL11A rs1427407 GG/GT genotypes appear to be associated with increased risks of VOC and stroke among SCD patients.

\section{DISCUSSION}

The BCL11A rs1427407 variant is commonly found in SCD patients. ${ }^{12)}$ The BCL11A gene was first detected in B-cell chronic lymphocytic leukemia and is a protooncogene for malignant hematological diseases. ${ }^{13,14)}$ Our study revealed that SCD patients had plasma BCL11A levels that were significantly higher than the control subjects $(100.13 \mu \mathrm{g} / \mathrm{L}$ versus $70.88 \mu \mathrm{g} / \mathrm{L}, \mathrm{P}<0.05)$. Furthermore, the SCD patients had a higher frequency of the GG and GT genotypes at BCL11A rs1427407. Moreover, the BCL11A rs1427407 GG variant increased the risk of developing SCD (Table 2). Some studies have also indicated that BCL11A can be used to predict overall and disease-free survivals. ${ }^{15,16)}$ In our study, the plasma BCL11A levels increased according to the duration of VOC in SCA patients. However, previous studies have revealed conflicting data regarding the associations between BCL11A variants and SCA-related complications, such as VOC frequency ${ }^{11,17)}$ and stroke. ${ }^{11,18)} \mathrm{We}$ observed statistically significant problems with the inheritance of BCL11A rs1427407 in >3 VOC/y or stroke, while the frequency of BCL11A rs1427407 genotypes was significantly related to vaso occlusive (VOc) periods in SCA patients (Table 3). Therefore, plasma BCL11A levels appear to be higher for more advanced VOC relative to the more initial stages (Figure 1). Our study also revealed that the BCL11A rs1427407 genotypes (GG/GT/TT) were significantly associated with stroke complications among SCD patients, and this finding agrees with the results of Satraf et al. ${ }^{19)}$ The presence of BCL11A rs1427407 appears to reflect a genetic hazard profile with a higher reticulocyte percentage and higher risk of stroke (Table 3 ). The association with VOC duration of $>3$ years also indicates that this genetic profile was strongly associated with markers of hemolysis and it appears that the presence of SCA is related to stroke complications. Our findings also agree with a previous report that a decreased hemoglobin level is a risk factor for stroke (Table 1), ${ }^{19)}$ and that increased markers of hemolysis are independently associated with increased reticulocytes and silent strokes. ${ }^{20)}$ Relative to the studies performed by Saraf et al. ${ }^{19)}$ and Kwiatkowski et al., ${ }^{21)}$ our study has several useful parameters. For example, regardless of the cross-sectional design, we evaluated variations in stroke history according to the BCL11A rs1427407 genotypes by retrospectively collecting stroke records that were prepared using a medical evaluation tool. This may have reduced the risk of detection bias and protected against including silent infarcts in this analysis, which increased the likelihood of identifying VOC and stroke in SCD patients.

In conclusion, the present study revealed that the BCL11A rs 1427407 genotypes and elevated BCL11A plasma levels were associated with the risk of stroke, which indicates that genetic factors influence the degree of hemolysis and HbF levels, which may improve our ability to identify SCA patients who are experiencing these complications. Furthermore, plasma BCL11A levels were elevated in patients with the GG/GT genotype of BCL11A rs1427407. To the best of our knowledge, this is the first study to evaluate the genetic influence on SCA severity among Sudanese patients, which may help improve the medical management of these cases. However, our findings must be validated by larger studies. Nevertheless, if further research confirms our results, our findings may indicate that genetic evaluations can help improve the treatment of patients with SCA who are experiencing stroke complications or VOC.

\section{CONFLICT OF INTEREST}

No potential conflict of interest relevant to this article was reported.

\section{ORCID}

Fathelrahman Mahdi Hassan: https://orcid.org/0000-0002-2630-3208 Faisal Mousa Al-zahrani: https://orcid.org/0000-0003-3354-1172

\section{REFERENCES}

1. Cheesman S. Sickle cell disease: symptoms, complications and management. Clin Pharm 2015;7. https://doi.org/10.1211/CP.2015.20069268.

2. Modell B, Darlison M. Global epidemiology of haemoglobin disorders and derived service indicators. Bull World Health Organ 2008;86:4807 .

3. Oniyangi O, Omari AA. Malaria chemoprophylaxis in sickle cell disease. Cochrane Database Syst Rev 2006;(4):CD003489.

4. Rees DC, Olujohungbe AD, Parker NE, Stephens AD, Telfer P, Wright J, et al. Guidelines for the management of the acute painful crisis in sickle cell disease. Br J Haematol 2003;120:744-52.

5. Pule GD, Ngo Bitoungui VJ, Chetcha Chemegni B, Kengne AP, Antonarakis S, Wonkam A. Association between variants at BCL11A erythroid-specific enhancer and fetal hemoglobin levels among sickle cell disease patients in Cameroon: implications for future therapeutic interventions. OMICS 2015;19:627-31. 
6. Thein SL, Menzel S. Discovering the genetics underlying foetal haemoglobin production in adults. Br J Haematol 2009;145:455-67.

7. Xu J, Peng C, Sankaran VG, Shao Z, Esrick EB, Chong BG, et al. Correction of sickle cell disease in adult mice by interference with fetal hemoglobin silencing. Science 2011;334:993-6.

8. Bauer DE, Kamran SC, Lessard S, Xu J, Fujiwara Y, Lin C, et al. An erythroid enhancer of BCL11A subject to genetic variation determines fetal hemoglobin level. Science 2013;342:253-7.

9. Sebastiani P, Farrell JJ, Alsultan A, Wang S, Edward HL, Shappell H, et al. BCL11A enhancer haplotypes and fetal hemoglobin in sickle cell anemia. Blood Cells Mol Dis 2015;54:224-30.

10. Saiki RK, Scharf S, Faloona F, Mullis KB, Horn GT, Erlich HA, et al. Enzymatic amplification of beta-globin genomic sequences and restriction site analysis for diagnosis of sickle cell anemia. Science 1985;230: $1350-4$.

11. Wonkam A, Ngo Bitoungui VJ, Vorster AA, Ramesar R, Cooper RS, Tayo B, et al. Association of variants at BCL11A and HBS1L-MYB with hemoglobin $\mathrm{F}$ and hospitalization rates among sickle cell patients in Cameroon. PLoS One 2014;9:e92506.

12. Bhanushali AA, Patra PK, Nair D, Verma H, Das BR. Genetic variant in the BCL11A (rs1427407), but not HBS1-MYB (rs6934903) loci associate with fetal hemoglobin levels in Indian sickle cell disease patients. Blood Cells Mol Dis 2015;54:4-8.

13. Nakamura T, Yamazaki Y, Saiki Y, Moriyama M, Largaespada DA, Jenkins NA, et al. Evi9 encodes a novel zinc finger protein that physically interacts with BCL6, a known human B-cell proto-oncogene product. Mol Cell Biol 2000;20:3178-86.

14. Weniger MA, Pulford K, Gesk S, Ehrlich S, Banham AH, Lyne L, et al.
Gains of the proto-oncogene BCL11A and nuclear accumulation of $\mathrm{BCL11A}(\mathrm{XL})$ protein are frequent in primary mediastinal B-cell lymphoma. Leukemia 2006;20:1880-2.

15. Jiang BY, Zhang XC, Su J, Meng W, Yang XN, Yang JJ, et al. BCL11A overexpression predicts survival and relapse in non-small cell lung cancer and is modulated by microRNA-30a and gene amplification. Mol Cancer 2013;12:61.

16. Zhang N, Jiang BY, Zhang XC, Xie Z, Su J, Zhang Q, et al. The BCL11A$\mathrm{XL}$ expression predicts relapse in squamous cell carcinoma and large cell carcinoma. J Thorac Dis 2015;7:1630-6.

17. Lettre G, Sankaran VG, Bezerra MA, Araujo AS, Uda M, Sanna S, et al. DNA polymorphisms at the BCL11A, HBS1L-MYB, and beta-globin loci associate with fetal hemoglobin levels and pain crises in sickle cell disease. Proc Natl Acad Sci U S A 2008;105:11869-74.

18. Leonardo FC, Brugnerotto AF, Domingos IF, Fertrin KY, de Albuquerque DM, Bezerra MA, et al. Reduced rate of sickle-related complications in Brazilian patients carrying HbF-promoting alleles at the BCL11A and HMIP-2 loci. Br J Haematol 2016;173:456-60.

19. Saraf SL, Akingbola TS, Shah BN, Ezekekwu CA, Sonubi O, Zhang X, et al. Associations of $\alpha$-thalassemia and BCL11A with stroke in Nigerian, United States, and United Kingdom sickle cell anemia cohorts. Blood Adv 2017;1:693-8.

20. Ohene-Frempong K, Weiner SJ, Sleeper LA, Miller ST, Embury S, Moohr JW, et al. Cerebrovascular accidents in sickle cell disease: rates and risk factors. Blood 1998;91:288-94.

21. Kwiatkowski JL, Zimmerman RA, Pollock AN, Seto W, Smith-Whitley $\mathrm{K}$, Shults J, et al. Silent infarcts in young children with sickle cell disease. Br J Haematol 2009;146:300-5. 